\title{
Gravity modes as a way to distinguish between hydrogen- and helium-burning red giant stars
}

\author{
Timothy R. Bedding ${ }^{1}$, Benoit Mosser ${ }^{2}$, Daniel Huber ${ }^{1}$, Josefina Montalbán $^{3}$, Paul Beck ${ }^{4}$, Jørgen Christensen-Dalsgaard ${ }^{5}$, \\ Yvonne P. Elsworth ${ }^{6}$, Rafael A. García ${ }^{7}$, Andrea Miglio ${ }^{3,6}$, Dennis Stello ${ }^{1}$, Timothy R. White ${ }^{1}$, Joris De Ridder ${ }^{4}$, Saskia Hekker ${ }^{6,8}$, \\ Conny Aerts ${ }^{4,9}$, Caroline Barban ${ }^{2}$, Kevin Belkacem ${ }^{10}$, Anne-Marie Broomhall ${ }^{6}$, Timothy M. Brown ${ }^{11}$, Derek L. Buzasi ${ }^{12}$, \\ Fabien Carrier ${ }^{4}$, William J. Chaplin ${ }^{6}$, Maria Pia Di Mauro ${ }^{13}$, Marc-Antoine Dupret ${ }^{3}$, Søren Frandsen ${ }^{5}$, Ronald L. Gilliland ${ }^{14}$, \\ Marie-Jo Goupil ${ }^{2}$, Jon M. Jenkins ${ }^{15}$, Thomas Kallinger ${ }^{16}$, Steven Kawaler ${ }^{17}$, Hans Kjeldsen ${ }^{5}$, Savita Mathur ${ }^{18}$, Arlette Noels ${ }^{3}$, \\ Victor Silva Aguirre ${ }^{19}$ \& Paolo Ventura ${ }^{20}$
}

Red giants are evolved stars that have exhausted the supply of hydrogen in their cores and instead burn hydrogen in a surrounding shell ${ }^{1,2}$. Once a red giant is sufficiently evolved, the helium in the core also undergoes fusion ${ }^{3}$. Outstanding issues in our understanding of red giants include uncertainties in the amount of mass lost at the surface before helium ignition and the amount of internal mixing from rotation and other processes ${ }^{4}$. Progress is hampered by our inability to distinguish between red giants burning helium in the core and those still only burning hydrogen in a shell. Asteroseismology offers a way forward, being a powerful tool for probing the internal structures of stars using their natural oscillation frequencies ${ }^{5}$. Here we report observations of gravity-mode period spacings in red giants ${ }^{6}$ that permit a distinction between evolutionary stages to be made. We use high-precision photometry obtained by the Kepler spacecraft over more than a year to measure oscillations in several hundred red giants. We find many stars whose dipole modes show sequences with approximately regular period spacings. These stars fall into two clear groups, allowing us to distinguish unambiguously between hydrogen-shell-burning stars (period spacing mostly $\sim 50$ seconds) and those that are also burning helium (period spacing $\sim 100$ to 300 seconds).

Oscillations in red giants, like those in the Sun, are thought to be excited by near-surface convection. The observed oscillation spectra are indeed remarkably Sun-like, with a broad range of radial and nonradial modes in a characteristic comb pattern ${ }^{7-11}$. However, theoretical models of red giants ${ }^{12-16}$ reveal a more complicated story for the nonradial modes (those with angular degree $l \geq 1$ ), and it has been suggested that this offers a means to determine the evolutionary states of these stars ${ }^{15}$. Owing to the large density gradient outside the helium core, a red giant is effectively divided into two cavities. In the envelope, the oscillations have properties of acoustic pressure modes ( $\mathrm{p}$ modes), but in the core, they behave like gravity modes (g modes), with buoyancy as the restoring force. The models predict a very dense spectrum of these so-called mixed modes for each value of $l$ (except $l=0$, as radial $g$ modes do not exist). Most mixed modes have a much larger amplitude in the core than in the envelope, and we refer to them as g-dominated mixed modes. Like pure $g$ modes, they are approximately equally spaced in period ${ }^{17,18}$, and measuring their average period spacing $(\Delta P)$ would give a valuable new asteroseismic probe of the cores of red giants. Unfortunately, they have very high inertias (the total interior mass that is affected by the oscillation), which leads to a very low amplitude at the stellar surface and makes them essentially impossible to observe. However, because of resonant coupling between the two cavities, some of the mixed modes have an enhanced amplitude in the envelope, making them more like $\mathrm{p}$ modes. These $\mathrm{p}$-dominated mixed modes have a lower inertia than the g-dominated mixed modes, and so their amplitudes can be high enough to render them observable. We expect their frequencies to be shifted from the regular asymptotic spacing, a feature known as 'mode bumping'19.

Figure 1a shows theoretical oscillation frequencies in a red giant of mass $1.5 M_{\odot}$ (where $M_{\odot}$ is the solar mass). The dashed lines show the radial modes $(l=0)$, whose frequencies decrease with time as the envelope of the star expands. These are pure $\mathrm{p}$ modes and are approximately equally spaced in frequency, with a separation of $\Delta v$. The solid lines show the much denser spectrum of dipole modes $(l=1)$. The g-dominated mixed modes appear as upward-sloping lines whose frequencies increase with time as the stellar core contracts. These modes are approximately equally spaced in period. The downward-sloping features that run parallel to the $l=0$ modes are produced by mode bumping: the p-dominated mixed modes, with frequencies decreasing with age, undergo avoided crossings ${ }^{19}$ with the g-dominated mixed modes. A similar pattern of mode bumping and avoided crossings is seen in models of subgiant stars ${ }^{13,20}$.

In Fig. $1 \mathrm{~b}$ we show the period spacings between adjacent $l=1$ modes in one of the models, indicated in Fig. 1a with the vertical line. The dips in Fig. $1 \mathrm{~b}$ correspond to bumped modes that are squeezed together. The period spacing of the g-dominated modes $\left(\Delta P_{\mathrm{g}}\right)$ can be measured from the upper envelope but cannot be observed directly because only the bumped modes have enough p-mode character to be detected, by virtue of their reduced mode inertias ${ }^{6,12-14}$. Observations will detect only a few modes in each p-mode order, and the average spacings of those observable sequences $\left(\Delta P_{\text {obs }}\right)$ will be less than the true g-mode spacing by up to a factor of two (the actual value depends on the number of modes detected, which is a function of the signal-tonoise ratio in the data, and on the strength of the coupling between the $\mathrm{g}$ - and $\mathrm{p}$-mode cavities $^{20}$ ). Figure $1 \mathrm{c}$ shows the mode frequencies of the model in Fig. $1 \mathrm{~b}$ displayed in échelle format, where the spectrum has been divided into segments that are stacked one above the other. Note

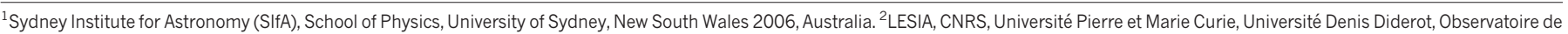
Paris, 92195 Meudon cedex, France. ${ }^{3}$ Institut d'Astrophysique et de Géophysique de I'Université de Liège, Allée du 6 Août 17, B 4000 Liège, Belgium. Instituut voor Sterrenkunde, KU Leuven,

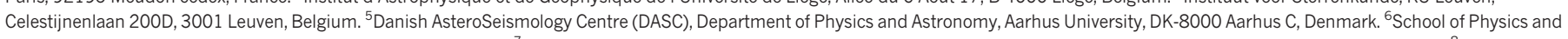

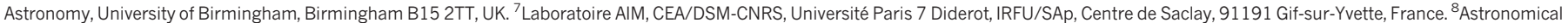

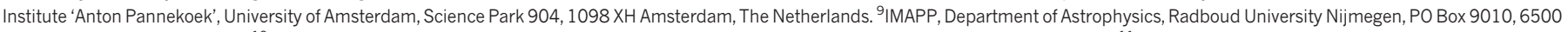
GL Nijmegen, The Netherlands. ${ }^{10}$ Institut d'Astrophysique Spatiale, UMR 8617, Université Paris XI, Bâtiment 121, 91405 Orsay Cedex, France. ${ }^{11}$ Las Cumbres Observatory Global Telescope, Goleta,

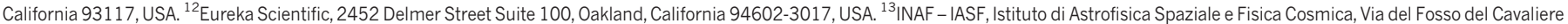

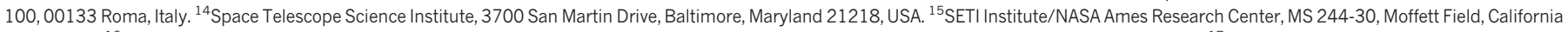

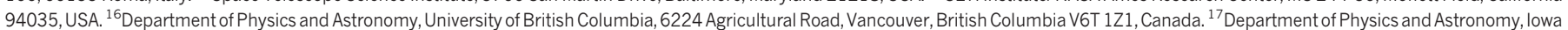
State University, Ames, lowa 50011, USA. ${ }^{18}$ High Altitude Observatory, NCAR, PO Box 3000, Boulder, Colorado 80307, USA. ${ }^{19}$ Max-Planck-Institut für Astrophysik, Karl-Schwarzschild-Str. 1, 85748 Garching, Germany. ${ }^{20}$ INAF - Osservatorio Astronomico di Roma, Via Frascati 33, 00040 Monte Porzio Catone (RM), Italy.
} 


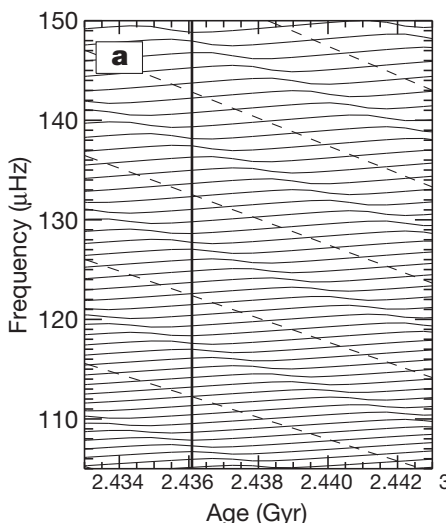

Age (Gyr)
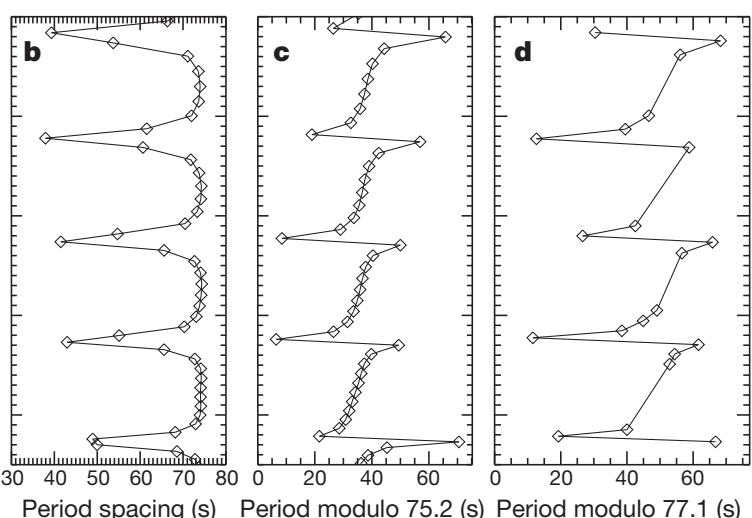

Figure $1 \mid$ Mixed modes and avoided crossings in red giant stars. a, Evolution with time of oscillation frequencies in a model of a hydrogen-shellburning red giant with a mass of $1.5 M_{\odot}$ and solar metallicity, calculated using the Aarhus stellar evolution code ASTEC ${ }^{27}$. Dashed lines show radial modes $(l=0)$ and solid lines show dipole modes $(l=1)$. The models span ranges in radius and luminosity of $6.3-6.7 R_{\odot}$ and $19.1-21.4 L_{\odot}$, respectively (here $R_{\odot}$ is the solar radius, and $L_{\odot}$ the solar luminosity). b, Period spacings between adjacent $l=1$ modes for the model marked with a vertical line in $\mathbf{a}$. The period spacing of the $\mathrm{g}$-dominated modes $\left(\Delta P_{\mathrm{g}}\right)$ can be seen from the maximum values to be about $75 \mathrm{~s}$. Note that model frequencies were not corrected for near-

that the abscissa shows the period modulo $\Delta P_{\mathrm{g}}$ (that is, the remainder after dividing by $\Delta P_{\mathrm{g}}$ ), whereas a conventional échelle diagram plots frequencies modulo the p-mode frequency spacing, $\Delta v$.

Sequences of $l=1$ modes with approximately constant period spacings were first observed in the red giant KIC 6928997 (ref. 6), and we have found similar patterns in several hundred more stars. The observations were obtained with the Kepler satellite over the first 13 months of its mission and were sampled every $29.4 \mathrm{~min}$ in the long-cadence mode ${ }^{21}$. Figure 1d shows the period échelle diagram for KIC 6928997 , and allows us to estimate the spacing of the g-dominated modes to be $\Delta P_{\mathrm{g}}=77.1 \mathrm{~s}$, which is the value required to produce a vertical alignment. Remarkably, we have been able to estimate $\Delta P_{\mathrm{g}}$ despite the fact that g-dominated modes are not observed (the average spacing of the observed modes ${ }^{6}$ is $\Delta P_{\text {obs }} \approx 55 \mathrm{~s}$ ).

Figure 2 compares observed power spectra of two red giants that have similar $\mathrm{p}$-mode spacings $(\Delta v \approx 8 \mu \mathrm{Hz})$ but very different $l=1$ period spacings. We note that the outermost peaks in each $l=1$ cluster surface effects ${ }^{6,28}$, which would have a small effect on the period spacings. c, Échelle diagram of $l=1$ modes for the same model as shown in $\mathbf{b}$. Here, the oscillation spectrum has been divided into segments of fixed length that are stacked one above the other. Note that the abscissa is the period modulo the g-mode period spacing, $\Delta P_{\mathrm{g}}$ (whereas a conventional échelle diagram plots frequencies modulo the p-mode frequency spacing, $\Delta v$ ). d, Échelle diagram of observed $l=1$ frequencies in the star KIC 6928997. We conclude that the true g-mode spacing is $\Delta P_{\mathrm{g}}=77.1 \mathrm{~s}$, whereas the average spacing of the observed modes $^{6}$ was found to be $\Delta P_{\text {obs }} \approx 55 \mathrm{~s}$.

(values of $l$ are given above the peaks), which we expect to be the closest in character to the g-dominated modes, appear to be the narrowest. This observation is consistent with theoretical calculations of mode inertias and lifetimes ${ }^{13-16}$. Once again, we have detected enough modes to determine $\Delta P_{\mathrm{g}}$ unambiguously using échelle diagrams (right panels). We find $\Delta P_{\mathrm{g}}$ for the two stars to differ by about a factor of two, implying they have very different core properties.

Inferring $\Delta P_{\mathrm{g}}$ in this way using the period échelle diagram is not possible for most of the stars in our sample, because it requires at least 3-4 modes to be detected in several of the $l=1$ clusters, which is only possible for the stars with the best signal-to-noise ratio. Therefore, we have instead measured the average period spacing of the observed $l=1$ modes $\left(\Delta P_{\text {obs }}\right)$ by using the power spectrum of the power spectrum. In this method, the power spectrum was first expressed in period rather than frequency and then set to zero in regions not containing power from the $l=1$ modes, as determined using the methods of ref. 10. The power spectrum of this power spectrum was then calculated in order to
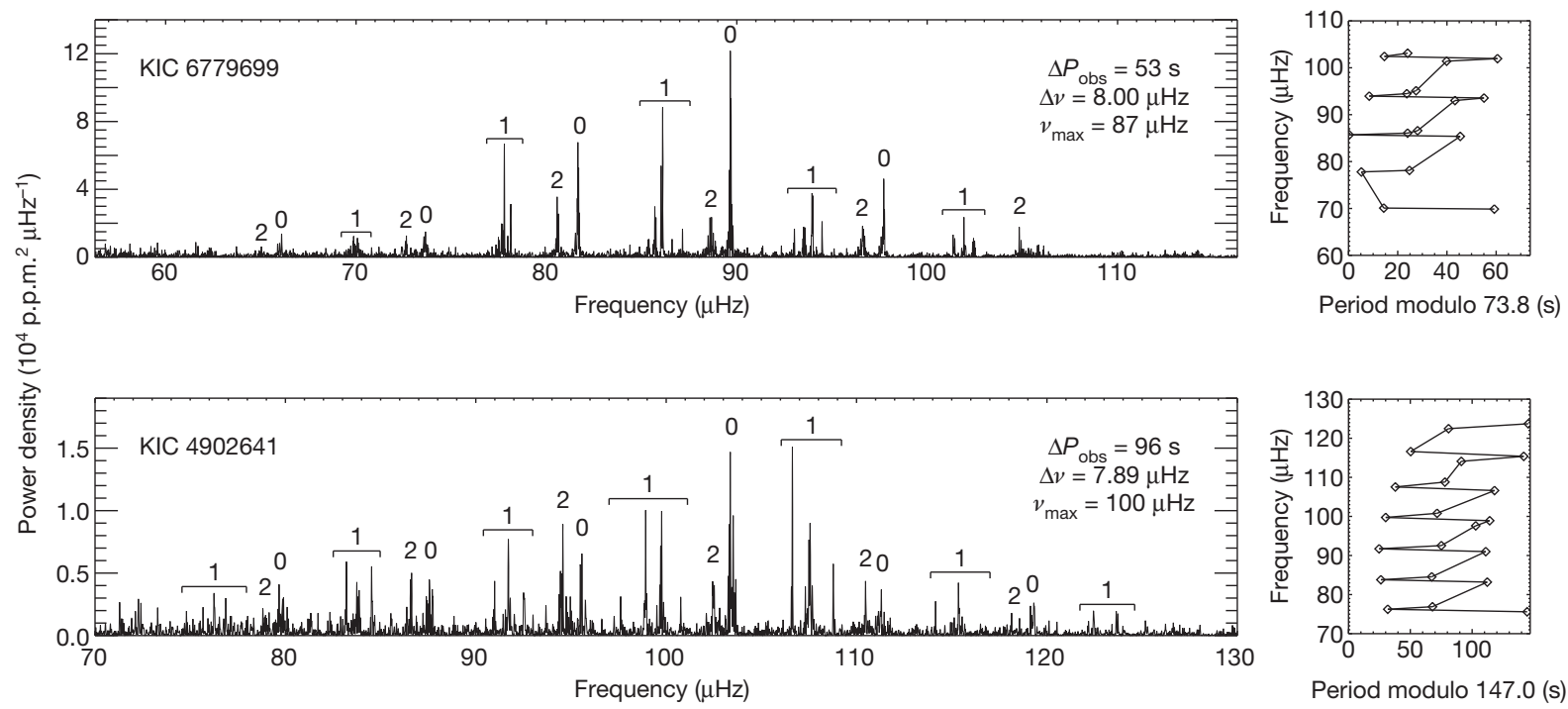

Figure 2 Oscillation power spectra and échelle diagrams of two red giant stars observed with Kepler. Top, KIC 6779699; bottom, KIC 4902641: left, power spectra; right, échelle diagrams. The difference in the spacings of the $l=1$ modes indicates that KIC 6779699 is undergoing hydrogen-shell-burning

on the red giant branch, while KIC 4902641 is also burning helium in its core (see Fig. 3). Observations of KIC 6779699 were made over the first 13 months of the Kepler mission (Q0-Q5, where Q indicates the quarter), while those of KIC 4902641 were made over the first 10 months (Q0-Q4). 
determine the most prominent period spacing. For the reasons mentioned above, we expect $\Delta P_{\text {obs }}$ to be less than $\Delta P_{\mathrm{g}}$ and we have measured the ratio between them to be in the range 1.3-1.6 in the few cases where $\Delta P_{\mathrm{g}}$ can be estimated unambiguously. Two other methods to

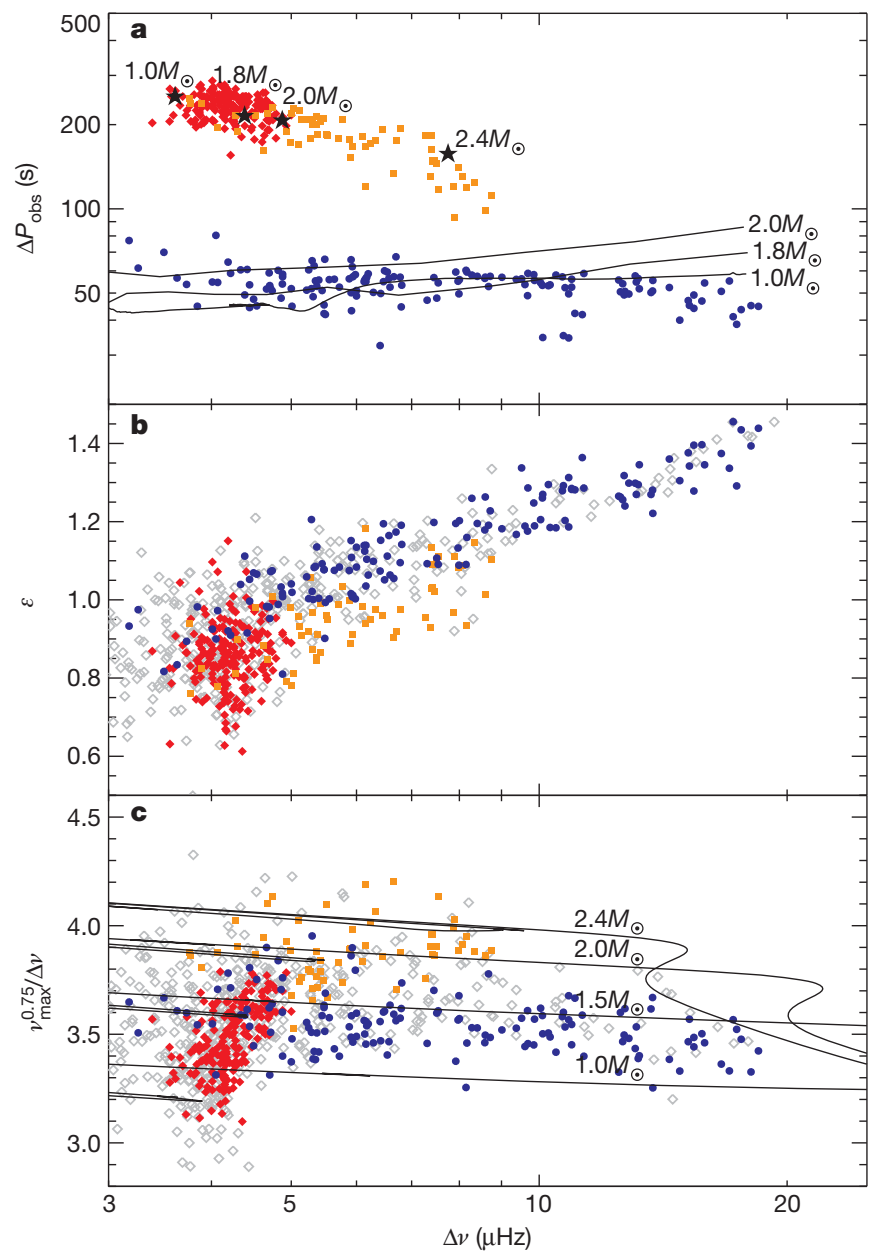

Figure $3 \mid$ Asteroseismic diagrams for red giants observed with Kepler. The abscissa is the p-mode large frequency separation. a, Filled symbols show period spacings measured from observations (Supplementary Table). The stars divide into two clear groups, with blue circles indicating hydrogen-shellburning giants (143 stars), while the remainder are helium-core-burning stars (193 red diamonds and 61 orange squares, divided on the basis of panel c). The solid lines show average observable period spacings for ASTEC ${ }^{27}$ models of hydrogen-shell-burning giants on the red giant branch as they evolve from right to left, calculated from the central three modes in the $l=1$ clusters. The black stars show theoretical period spacings calculated in the same way, for four models of helium-core-burning stars that are midway through that phase (core helium fraction 50\%). The 2.4- $M_{\odot}$ model was calculated with ASTEC $^{27}$ and commenced helium-burning without passing through a helium flash. The other three models, which did undergo a helium flash, were computed using the ATON $\operatorname{code}^{15,29}$ (J.M. et al., manuscript in preparation). Solar metallicity was adopted for all models, which were computed without mass loss. $\mathbf{b}$, The quantity $\varepsilon$, which specifies the absolute frequency of the p-mode comb pattern $^{10,11}$. We see a systematic difference between the hydrogen- and heliumburning stars. The open grey diamonds are stars for which a reliable period spacing could not be measured (391 stars). Many of these had high signal-tonoise but lacked a clear structure that would indicate regular period spacings. c, The quantity $v_{\max }{ }^{0.75} / \Delta v$, which is approximately independent of luminosity ${ }^{10}$ (here $v_{\max }$ and $\Delta v$ are in $\mu \mathrm{Hz}$ ). Helium-burning stars that we identify as belonging to the red clump, based on their positions in this diagram, are marked with red diamonds. The remainder, which presumably belong to the secondary clump, are marked with orange squares. The lines show model calculations based on scaling relations ${ }^{26}$ for $\Delta v$ and $v_{\max }$ applied to solarmetallicity BASTI models ${ }^{30}$ with the mass loss efficiency parameter set to $\eta=0.2$. measure $\Delta P_{\text {obs }}$ gave comparable results. The first was simply to measure pairwise separations of the strongest $l=1$ peaks in the power spectrum. The other was to calculate the autocorrelation of the time series $^{22}$ with narrow filters centred on the $l=1$ clusters.

Observed period spacings for about 400 stars are shown in Fig. 3a, which clearly demonstrates the existence of two distinct populations with different core properties. A comparison with model calculations confirms that the two groups coincide with hydrogen-shell-burning stars on the red giant branch (blue circles) and those that are also burning helium in the core (red diamonds and orange squares). We conclude that $\Delta P_{\mathrm{obs}}$ is an extremely reliable parameter for distinguishing between stars in these two evolutionary stages, which are known to have very different core densities ${ }^{15}$ but are otherwise very similar in their fundamental properties (mass, luminosity and radius). We note that other asteroseismic observables, such as the small p-mode separations, are not able to do this ${ }^{10,15}$.

Our ability to distinguish between hydrogen- and helium-burning stars makes it possible to investigate their properties as separate populations. One example is the parameter $\varepsilon$, which specifies the absolute position of the p-mode comb pattern ${ }^{10,11}$. As shown in Fig. $3 \mathrm{~b}$, there is a systematic offset between the two populations. This may indicate a difference in the surface layers, given that $\varepsilon$ is sensitive to the upper turning point of the modes ${ }^{23}$. However, the difference may also arise because the envelope of oscillation power is centred at different frequencies in the two types of stars (see below). This result is clearly worthy of further study.

A very important application for the helium-burning stars is to distinguish between the so-called red clump and the secondary clump $p^{24,25}$. The red clump comprises low-mass stars that suffered from electron degeneracy in their hydrogen-shell-burning phase and ignited helium in a flash (that is, all the helium ignited more or less simultaneously) once the core attained a critical mass. This common core mass explains why the red clump (known as the horizontal branch when seen in metal-poor clusters) spans a very narrow range of luminosities. The secondary-clump stars, meanwhile, are too massive to have undergone a helium flash and so have a range of core masses, and hence of luminosities. The mass threshold that divides these two populations depends on metallicity, and also on core overshoot ${ }^{24}$ (the phenomenon in which the motion of convection cells extends beyond the nominal boundary of the convective zone).

Among the helium-burning stars in Fig. 3a we can indeed see this division into a compact group (the red clump; red diamonds) and a dispersed group (the secondary clump; orange squares). It is even more apparent when we examine the quantity $v_{\max } 0.75 / \Delta v$, which is approximately independent of luminosity ${ }^{10}\left(v_{\max }\right.$ is the frequency at which the oscillation envelope has its maximum $\left.{ }^{26}\right)$. This quantity is shown in Fig. $3 c$, and the comparison with evolutionary models having solar metallicity implies a helium-flash threshold of around $2 M_{\odot}$. Refinement of this result, using data from more detailed studies of individual stars near the boundary between the red clump and the secondary clump, should test predictions of convective-core overshoot.

\section{Received 24 December 2010; accepted 16 February 2011.}

1. Schwarzschild, M. \& Härm, R. Red giants of population II. II. Astrophys. J. 136, 158-165 (1962).

2. Iben, I. Jr. Low-mass red giants. Astrophys. J. 154, 581-595 (1968),

3. Sweigart, A. V.\& Gross, P. G. Evolutionary sequences for red giant stars. Astrophys. J. Suppl. Ser. 36, 405-437 (1978).

4. Charbonnel, C. in Cosmic Abundances as Records of Stellar Evolution and Nucleosynthesis (eds Barnes, T. G. \& Bash, F. N.) 119-130 (Vol. 336, Astronomical Society of the Pacific Conference Series, 2005).

5. Aerts, C., Christensen-Dalsgaard, J. \& Kurtz, D. W. Asteroseismology (Springer, 2010).

6. Beck, P. G. etal. Detection of gravity-mode period spacings in red giant stars by the Kepler Mission. Science (in the press).

7. De Ridder, J. et al. Non-radial oscillation modes with long lifetimes in giant stars. Nature 459, 398-400 (2009).

8. Kallinger, T.etal. Oscillating red giants in the CoRoT exo-field: asteroseismic radius and mass determination. Astron. Astrophys. 509, A77 (2010). 
9. Bedding, T. R. et al. Solar-like oscillations in low-luminosity red giants: first results from Kepler. Astrophys. J. 713, L176-L181 (2010).

10. Huber, D. et al. Asteroseismology of red giants from the first four months of Kepler data: global oscillation parameters for 800 stars. Astrophys. J. 723, 1607-1617 (2010).

11. Mosser, B. et al. The universal red-giant oscillation pattern. An automated determination with CoRoT data. Astron. Astrophys. 525, L9 (2011).

12. Dziembowski, W. A., Gough, D. O., Houdek, G. \& Sienkiewicz, R. Oscillations of $\alpha$ UMa and other red giants. Mon. Not. R. Astron. Soc. 328, 601-610 (2001).

13. Christensen-Dalsgaard, J. Physics of solar-like oscillations. Sol. Phys. 220, 137-168 (2004).

14. Dupret, M. et al. Theoretical amplitudes and lifetimes of non-radial solar-like oscillations in red giants. Astron. Astrophys. 506, 57-67 (2009).

15. Montalbán, J., Miglio, A., Noels, A., Scuflaire, R. \& Ventura, P. Seismic diagnostics of red giants: first comparison with stellar models. Astrophys. J. 721, L182-L188 (2010).

16. Di Mauro, M. P. etal. Solar-like oscillations from the depths of the red-giant star KIC 4351319 observed with Kepler. Mon. Not. R. Astron. Soc. (submitted).

17. Tassoul, M. Asymptotic approximations for stellar nonradial pulsations. Astrophys. J. Suppl. Ser. 43, 469-490 (1980).

18. Miglio, A., Montalbán, J., Eggenberger, P. \& Noels, A. Gravity modes and mixed modes as probes of stellar cores in main-sequence stars: from solar-like to $\beta$ Cep stars. Astron. Nachr. 329, 529-534 (2008).

19. Aizenman, M., Smeyers, P. \& Weigert, A. Avoided crossing of modes of non-radial stellar oscillations. Astron. Astrophys. 58, 41-46 (1977).

20. Deheuvels, S. \& Michel, E. New insights on the interior of solar-like pulsators thanks to CoRoT: the case of HD 49385. Astrophys. Space Sci. 328, 259-263 (2010).

21. Jenkins, J. M. et al. Initial characteristics of Kepler long cadence data for detecting transiting planets. Astrophys. J. 713, L120-L125 (2010).

22. Mosser, B. \& Appourchaux, T. On detecting the large separation in the autocorrelation of stellar oscillation times series. Astron. Astrophys. 508, 877-887 (2009).

23. Gough, D. O. in Hydrodynamic and Magnetodynamic Problems in the Sun and Stars (ed. Osaki, Y.) 117-143 (Univ. Tokyo Press, 1986).

24. Girardi, L. A secondary clump of red giant stars: why and where. Mon. Not. R. Astron. Soc. 308, 818-832 (1999).

25. Miglio, A. et al. Probing populations of red giants in the galactic disk with CoRoT. Astron. Astrophys. 503, L21-L24 (2009).
26. Kjeldsen, H. \& Bedding, T. R. Amplitudes of stellar oscillations: the implications for astero-seismology. Astron. Astrophys. 293, 87-106 (1995).

27. Christensen-Dalsgaard, J. ASTEC - the Aarhus STellar Evolution Code. Astrophys. Space Sci. 316, 13-24 (2008)

28. Kjeldsen, H., Bedding, T. R. \& Christensen-Dalsgaard, J. Correcting stellar oscillation frequencies for near-surface effects. Astrophys. J. 683, L175-L178 (2008).

29. Ventura, P., D'Antona, F. \& Mazzitelli, I. The ATON 3.1 stellar evolutionary code. A version for asteroseismology. Astrophys. Space Sci. 316, 93-98 (2008).

30. Pietrinferni, A., Cassisi, S., Salaris, M. \& Castelli, F. A large stellar evolution database for population synthesis studies. I. Scaled solar models and isochrones. Astrophys. J. 612, 168-190 (2004).

Supplementary Information is linked to the online version of the paper at www.nature.com/nature.

Acknowledgements We acknowledge the entire Kepler team, whose efforts made these results possible. We thank M. Biercuk for comments. Funding for this Discovery mission was provided by NASA's Science Mission Directorate. T.R.B and D.S. were supported by the Australian Research Council; P.B. and C.A. were supported by European Community's 7th Framework Programme (PROSPERITY); S.H. was supported by the Netherlands Organisation for Scientific Research (NWO). The National Center for Atmospheric Research is sponsored by the National Science Foundation.

Author Contributions T.R.B, B.M., P.B., Y.P.E, R.A.G., S.H., C.A., A.-M.B. and F.C. measured and interpreted period spacings; B.M., D.H., R.A.G., S.H., T.K., W.J.C., C.B.,

D.L.B. and S.M. calculated power spectra and measured large frequency separations; J.M., J.C.-D., A.M., D.S., T.R.W., K.B., M.P.D.M., M.-A.D., M.-J.G., S.K., A.N., V.S.A. and P.V. calculated and interpreted theoretical models; J.D.R., S.H., S.F., Y.P.E., D.S., T.M.B., H.K J.C.-D. and R.L.G contributed to the coordination of the project, including the acquisition and distribution of the data; and J.M.J. constructed the photometric time series from the original Kepler pixel data. All authors discussed the results and commented on the manuscript.

Author Information Reprints and permissions information is available at www.nature.com/reprints. The authors declare no competing financial interests. Readers are welcome to comment on the online version of this article at www.nature.com/nature. Correspondence and requests for materials should be addressed to T.R.B. (t.bedding@physics.usyd.edu.au). 